\title{
ON THE THEOREM OF GLEASON AND MARSH ${ }^{1}$
}

\section{NEAL ZIERLER ${ }^{2}$}

Let $K$ be a field with a finite number $q$ of elements and let $\alpha$ be the mapping of $K[x]$ in itself that assigns

$$
f^{\alpha}(x)=\sum_{i=0}^{n} f_{i} x^{q^{i}-1}
$$

as image to

$$
f(x)=\sum_{i=0}^{n} f_{i} x^{i}
$$

The order of a nonzero element $a$ of a finite field is the smallest of the positive integers $j$ for which $a^{j}=1$. If $f(x)$ is irreducible over $K$, then all of its roots are of the same order, for given any two roots of $f$ lying in a finite extension $F$ of $K$ there is always an automorphism of $F$ mapping one on the other. We may therefore define the order of the irreducible polynomial $f$ to be the order of any one of its roots. The purpose of this note is to establish the following generalization of the theorem of Gleason and Marsh. ${ }^{3}$

THEOREM. Let $f$ be an irreducible member of $K[x]$. Then the degree of every irreducible factor of $f^{\alpha}$ is equal to the order of $f$.

Proof. Let $\beta$ be the mapping of $K[x]$ in itself such that $g^{\beta}(x)$ $=x g^{\alpha}(x)=\sum_{i=0}^{n} g_{i} x^{q^{i}}$. Clearly $\beta$ is linear over $K$; that is, if $g$ and $h$ are in $K[x]$ and $a$ and $b$ are in $K$ then $(a g+b h)^{\beta}=a g^{\beta}+b h^{\beta}$.

Let $g \in K[x]$. Then $(x g(x))^{\beta}=\sum g_{i} x^{q^{i+1}}=\left(\sum g_{i} x^{q^{i}}\right)^{q}=\left(g^{\beta}(x)\right)^{q}$. That is,

$$
(x g)^{\beta}=g^{\beta q} .
$$

Let $f, g$ and $a$ be in $K[x]$ and suppose $g=a f$. Then $g^{\beta}(x)$ $=\left(\sum a_{i} x^{i} f(x)\right)^{\beta}=\sum a_{i}\left(x^{i} f(x)\right)^{\beta}=\sum a_{i}\left(f^{\beta}(x)\right)^{i}$ by (1). Thus, $f^{\beta} \mid g^{\beta}$ and so $f^{\alpha} \mid g^{\alpha}$. This proves

$$
f \mid g \text { implies } f^{\alpha} \mid g^{\alpha} \text {. }
$$

Received by the editors August 12, 1957.

1 The research in this document was supported jointly by the Army, Navy, and Air Force under contract with the Massachusetts Institute of Technology.

2 Staff Member, Lincoln Laboratory, Massachusetts Institute of Technology.

${ }^{3}$ A. A. Albert, Fundamental concepts of higher algebra, University of Chicago Press, 1956, p. 132. 
Now let $f$ be irreducible, let $g$ be arbitrary and let $h$ be a factor of $f^{\alpha}$ of positive degree. We shall show that

$$
h \mid g^{\alpha} \text { implies } f \mid g \text {. }
$$

Let $A=\left\{b \in K[x]: h \mid b^{\alpha}\right\}$. If $b \in A$ and $a \in K[x], b^{\alpha} \mid(a b)^{\alpha}$ by and so $a b \in A$. It follows easily that $A$ is an ideal containing $f$ but not 1 in $K[x]$. Hence, since $f$ is irreducible and $K[x]$ is a principal ideal domain, $A=(f)$ and (3) is established.

Now let $f$ be irreducible of order $r$ and let $d$ be the degree of an irreducible factor $h$ of $f^{\alpha}$. Then $f \mid 1-x^{r}$ and it follows from (2) that $h \mid 1-x^{q^{r}-1}$. Hence a splitting field of $h$, which has $q^{d}$ elements, may be regarded as a subfield of a splitting field of $1-x^{q^{r}-1}$, which has $q^{r}$ elements, and so $d \mid r$. On the other hand, $h \mid 1-x^{q^{d}-1}$ implies $f \mid 1-x^{d}$ by (3) and hence $r \mid d$. It follows now that $d=r$ and the proof of the theorem is complete.

Corollary (Gleason-MaRsh). Let $f$ be an irreducible polynomial of degree $n$ over $K$. The order of $f$ is $q^{n}-1$ if and only if $f^{\alpha}$ is irreducible.

Massachusetts Institute of Technology 